\title{
Rice-derived rotavirus antibody shows promise
}

Researchers have genetically engineered rice to produce high yields of a rotavirus-specific antibody fragment in the seeds. The study, published in the Journal of Clinical Investigation, demonstrated the effectiveness of the antibody in treating and preventing rotavirus-induced gastrointestinal symptoms in mice. This plant-based platform could potentially be used to efficiently produce other biological therapeutics.

Yuki and colleagues harnessed findings from two previous studies to generate the antibody. In one previous study, llamas were immunized with the G3 strain of rhesus rotavirus and the resulting antibody fragment (named ARP1) was able to neutralize a broad range of rotavirus serotypes and genotypes. Llama antibodies are particularly attractive to use as biological therapeutics as the variable domain of the heavy chain has a high binding capacity and is resistant to pepsin and heat. Moreover, the small size and simple structure of the llama antibodies means that they are uncomplicated to produce as recombinant proteins. In the other study, a rice-based cholera toxin vaccine was effective in mice, thus providing proof of concept that rice can be genetically engineered to produce efficacious oral vaccines.

In the most recent study, the authors used the bacteria Agrobacterium tumefaciens to transfect a vector encoding the $A R P 1$ gene into a Japonica variety of rice plant. The vector also included an RNA interference (RNAi) cassette to suppress the plant's internal protein stores to maximize the storage of ARP1. Using this technology, the transgenic rice yielded an average of $170 \mu \mathrm{g}$ of ARP1 antibodies (named MucoRice-ARP1) per seed. By contrast, in seeds without the RNAi suppression, yields dropped to $14 \mu \mathrm{g}$.

In a mouse pup model of rotavirus infection, MucoRice-ARP1 attenuated disease symptoms when it was fed to the mice before or after rotavirus inoculation. Remarkably, MucoRice-ARP1 that was heattreated (boiled for either 10 minutes or 30 minutes at $95^{\circ} \mathrm{C}$ ) or stored at room temperature for more than a year retained its therapeutic and prophylactic properties to the same degree as freshly harvested MucoRice-ARP1.

On the basis of these results, the authors propose that this plantbased antibody platform could be a highly cost-efficient method for producing biological therapeutics, particularly in developing countries, as it dispenses with the need for cold storage and purification. The authors aim to initiate Phase I studies within 2 years.

Man Tsuey Tse

ORIGINAL RESEARCH PAPER Tokuhara, D. et al. Rice-based oral antibody fragment prophylaxis and therapy against rotavirus infection.J. Clin. Invest. 123, 3829-3838 (2013)

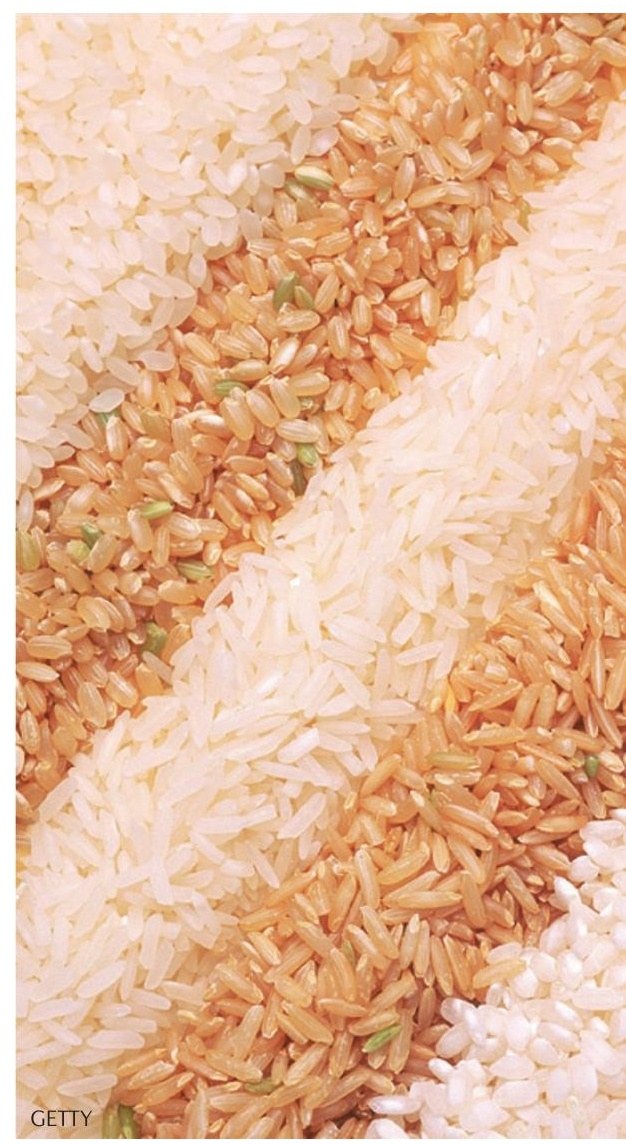

